\title{
Proof of principle for effectiveness of a gonorrhoea vaccine
}

\author{
Magnus Unemo and Aleksandra E. Sikora
}

\begin{abstract}
Antimicrobial resistance in Neisseria gonorrhoeae compromises gonorrhoea treatment globally and vaccines might be the only sustainable solution for gonorrhoea control. A new study for the first time provides a proof of principle for protection with $\sim 31 \%$ effectiveness against gonorrhoea, owing to cross-protection by the outer membrane vesicle Neisseria meningitidis serogroup B vaccine (MeNZB).
\end{abstract}

\begin{abstract}
Refers to Petousis-Harris, H. et al. Effectiveness of a group B outer membrane vesicle meningococcal vaccine against gonorrhoea in New Zealand: a retrospective case-control study. Lancet http://dx.doi.org/10.1016/ S0140-6736(17)31449-6 (2017)
\end{abstract}

Gonorrhoea, which is caused by Neisseria gonorrhoeae (gonococcus), is a major public health priority worldwide and 78 million cases among adults were estimated in 2012, predominantly in resource-poor settings ${ }^{1}$. Gonorrhoea is frequently asymptomatic, particularly in women. The severe complications of untreated infection include pelvic inflammatory disease, ectopic pregnancy, infertility, neonatal complications, and increased transmission and acquisition of $\mathrm{HIV}^{2-4}$.

Antimicrobial therapy, combined with prevention, diagnosis, partner notification, and epidemiological surveillance, is the mainstay of gonorrhoea management. However, N. gonorrhoeae has developed resistance to all antimicrobials introduced for empirical treatment ${ }^{3,4}$. Thus, enhanced antimicrobial resistance surveillance, rapid point-of-care diagnostic tests that ideally simultaneously predict resistance, and new antimicrobials are crucial for maintaining treatability of gonorrhoea ${ }^{2-4}$. Nevertheless, an effective gonococcal vaccine is probably the only sustainable solution for controlling gonorrhoea and substantially reducing the global public-health burden, owing to the rapid emergence of resistance to all introduced therapeutic antimicrobials, the predominance of gonorrhoea in resource-poor settings where syndromic treatment of symptomatic patients dominates, and the lack of symptoms in many patients. This notion has also been strongly emphasized by the WHO, other public health organizations, and researchers ${ }^{2-5}$.

Unfortunately, the few clinical trials of gonorrhoea vaccines that have been performed (all before 1990) were disappointing. The vaccines did not provide significant protection from infection ${ }^{6}$. Subsequent work has identified critical challenges to development of an effective gonococcal vaccine: high level of heterogeneity and variability of selected gonococcal antigens; lack of known correlates of protection; weak, mainly local, and transient immune response without immunological memory; gonococcal evasion of immune responses; and a lack of laboratory animal models that closely mimic human infection and disease $\mathrm{e}^{5,6}$. These complications resulted in pessimism for the development of an effective prophylactic gonorrhoea vaccine and low investment in this research field in the past two to three decades.

In a new report, Petousis-Harris et al. ${ }^{7}$ provide a proof of principle for vaccine protection against gonorrhoea. The researchers assessed the effectiveness of the outer membrane vesicle (OMV) Neisseria meningitidis serogroup B vaccine (MeNZB) against gonorrhoea in a retrospective case-control study that included young adults attending sexual-health clinics in New Zealand. MeNZB was administered to $>1$ million New Zealanders in response to a meningococcal epidemic during $2004-2008$ and $81 \%$ of the population aged $<20$ years were vaccinated in 2004-2006 (REF. 7). The vaccinated individuals were significantly less likely to have been diagnosed with gonorrhoea compared with controls (adjusted OR 0.69, 95\% CI $0.61-0.79 ; P<0.0001$ ), particularly in the years during and immediately after vaccine administration. The effectiveness of MeNZB against gonorrhoea was estimated to be $31 \%$ (95\% CI 21-39\%), after adjustments for ethnicity, deprivation, geographical area, and $\operatorname{sex}^{7}$. The level of effectiveness might not initially seem impressive; however, these findings are seminal, as they show for the first time that an OMV vaccine can have a substantial effect on a mucosal infection and, most importantly, that a vaccine can offer protection against gonorrhoea.

\section{C...these findings are}

seminal, as they show ... that a vaccine can offer protection against gonorrhoea.

Furthermore, mathematical modelling suggests that a vaccine that has only $30 \%$ efficacy could decrease gonorrhoea prevalence by $>30 \%$ within 15 years, if population coverage is sufficiently high and immunity is maintained $^{7}$. MeNZB is no longer available, but the licensed, four-component meningococcal serogroup B vaccine (4CMenB; BEXSERO; GlaxoSmithKline) includes the same OMV as MeNZB and three recombinant vaccine antigens (neisserial heparin-binding antigen (NHBA), factor $\mathrm{H}$-binding protein (fHbp), and Neisseria adhesin A (NadA)). Consequently, widespread administration of the $4 \mathrm{CMenB}$ to adolescents might also reduce gonorrhoea prevalence owing to cross-protection ${ }^{7}$.

Interestingly, the first proof of vaccine protection against gonorrhoea is not made with a gonococcal vaccine but, owing to 


\section{Box 1 | The global sexually transmitted infection (STI) vaccine roadmap ${ }^{5}$}

"[...] first, obtaining better epidemiologic data on infection and sequelae; second, modelling the theoretical impact of STI vaccines; third, advancing basic science and translational research; fourth, defining preferred product characteristics (PPCs); fifth, facilitating clinical evaluation and vaccine introduction; and finally, encouraging investment in STI vaccine development".

cross-protection, with a vaccine for N. meningitidis, which is genetically and antigenically closely related to $N$. gonorrhoeae. Research to elucidate the specific or unspecific antigens and mechanisms involved in the MeNZBmediated protection against gonorrhoea is crucial. Findings from such studies can guide the development of an effective gonococcal vaccine and now is the ideal time to strongly reinvigorate interest and investment in this area. Accordingly, many of the previously identified challenges might now be overcome. Since the failed early trials, we have considerably improved our understanding of gonococcal pathogenesis ${ }^{5,6,8-10}$. Studies have provided detailed information on some of the gonococcal vaccine candidate antigens, which might ideally be used in combination, for example targeting different essential steps or pathways of the pathogenesis. High-throughput technologies, such as genome sequencing, transcriptomics, and proteomics, have been used to identify and appropriately examine novel antigens and their diversity, variability, and expression. Our understanding of immune responses has also improved, including that of suppressive responses related to gonorrhoea and how to overcome them. Protection against reinfection with homologous and heterologous N. gonorrhoeae strains after immunization with gonococcal OMV and a T helper 1 cell adjuvant has been shown in mice. In addition, sophisticated mouse models, an experimental male-volunteer urethral gonorrhoea model, and many new vaccine and adjuvant technologies have been developed ${ }^{5,6,8-10}$.

Now, research efforts should be intensified in several areas: further exploration of gonococcal molecular pathogenesis (for example, the natural course of infection in different anatomical sites); simultaneous evaluation of multiple antigens (likely to be applicable for a polyvalent vaccine and possibly including OMV); elucidation of immune responses (for long-term protection and limited suppressive response in men and women); identification of surrogate measures for assessing immunity; improvement and enhanced availability of harmonized and quality-assured assays, as well as preclinical and clinical models; and identification of appropriate adjuvants and ideal vaccination routes. We also need to consider additional questions to achieve effective gonorrhoea vaccination, such as essential end points (inhibition of transmission, infection and/or disease and sequelae), appropriate target populations, evaluation and/or modelling of effects on burden of gonococcal infections and sequelae and cost-effectiveness, and enhanced advocacy and social marketing for acceptance of vaccination to achieve high coverage. A global roadmap ${ }^{5}$ to accelerate the development of vaccines for gonorrhoea and other sexually transmitted infections has already been developed and outlines the key action steps (BOX 1).

In conclusion, most essential components for development of an effective gonococcal vaccine might already be attainable and now serious international multidisciplinary commitments involving coordination, collaboration, communication, and financial investment are required. Public health organizations, clinicians, and researchers should place increasing emphasis on the new opportunities for developing an effective gonococcal vaccine, the most effective strategies, and lessons learnt from the developments of the meningococcal serogroup B vaccines, instead of focusing on the obstacles identified in previous decades.
Magnus Unemo is at the WHO Collaborating Centre for Gonorrhoea and other STIs, Department of Laboratory Medicine, Faculty of Medicine and Health, Örebro University Hospital and Örebro University, S. Grevrosengatan 2, SE-701 85 Örebro, Sweden.

Aleksandra E. Sikora is at the Department of Pharmaceutical Sciences, College of Pharmacy, Oregon State University, Corvallis, Oregon 97330, USA.

Correspondence to M.U. magnus.unemo@regionorebrolan.se doi:10.1038/nrurol.2017.139 Published online 31 Aug 2017

1. Newman, L. et al. Global estimates of the prevalence and incidence of four curable sexually transmitted infections in 2012 based on systematic review and global reporting. PLOS ONE 10, e0143304 (2015).

2. World Health Organization. Global action plan to control the spread and impact of antimicrobial resistance in Neisseria gonorrhoeae. WHO http://www.who.int/reproductivehealth/publications/ rtis/9789241503501/en/ (2012).

3. Wi, T. et al. Antimicrobial resistance in Neisseria gonorrhoeae: global surveillance and a call for international collaborative action. PLoS Med. 14, e1002344 (2017).

4. Unemo, M. \& Shafer, W. M. Antimicrobial resistance in Neisseria gonorrhoeae in the 21 st century: past, evolution, and future. Clin. Microbiol. Rev. 27, 587-613 (2014)

5. Gottlieb, S. L. \& Johnston, C. Future prospects for new vaccines against sexually transmitted infections. Curr. Opin. Infect. Dis. 30, 77-86 (2017).

6. Edwards, J. L., Jennings, M. P., Apicella, M. A. \& Seib, K. L. Is gonococcal disease preventable? The importance of understanding immunity and pathogenesis in vaccine development. Crit. Rev. Microbiol. 42, 928-941 (2016).

7. Petousis-Harris, H. et al. Effectiveness of a group B outer membrane vesicle meningococcal vaccine against gonorrhoea in New Zealand: a retrospective case-control study. Lancet http://dx.doi.org/10.1016/ S0140-6736(17)31449-6 (2017).

8. Zielke, R. A. et al. Proteomics-driven antigen discovery for development of vaccines against gonorrhea. Mol. Cell. Proteomics 15, 2338-2355 (2016). 9. Jerse, A. E. \& Deal, C. D. Vaccine research for gonococcal infections: where are we? Sex. Transm. Infect. 89 (Suppl. 4), iv63-iv68 (2013).

10. Liu, Y. et al. Experimental vaccine induces Th1-driven immune responses and resistance to Neisseria gonorrhoeae infection in a murine model. Mucosal Immunol. http://dx.doi.org/10.1038/mi.2017.11 (2017).

\section{Acknowledgements}

Work at the WHO Collaborating Centre for Gonorrhoea and other STIs is supported by grants to M.U. from the Research Committee of Örebro County and the Örebro University Hospital Foundation, Örebro, Sweden. Funding to A.E.S. was provided by grant R01-Al117235 from the National Institute of Allergy $\&$ Infectious Diseases NIH.

Competing interests statement

The authors declare no competing interests. 